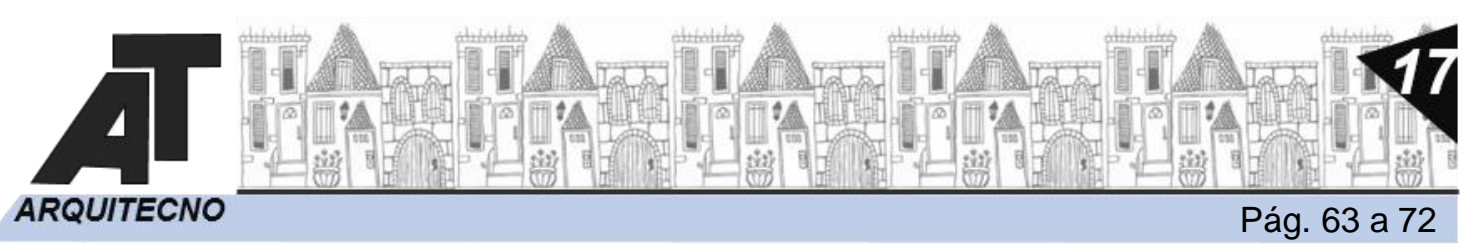

\title{
Análisis de condiciones ambiental en nuevas escuelas de clima cálido-húmedo, Tucumán, Argentina
}

\section{Environmental conditions analysis in new schools in hot and humid climate, Tucumán, Argentina}

\author{
Sandra Gabriela Marquez Vega y Cecilia Fernanda Martinez
}

Centro de Estudios Energía, Hábitat y Arquitectura Sustentable, Institut de Acondicionamiento Ambiental, Facultad de Arquitectura, Universidad Nacional de Tucumán, Argentina

sgmarquezvega@herrera.unt.edu.ar cmartinez@herrera.unt.edu.ar

\section{RESUMEN}

El trabajo analiza las condiciones ambientales en los espacios áulicos de escuelas públicas de la provincia de Tucumán, Argentina, construidas en el período 2003-2015. Se estudia el edificio prototípico, $2500 \mathrm{~m}^{2}$ promedio, verificando su adecuación a las estrategias bioclimáticas para la zona, evaluando el comportamiento térmico de la envolvente, determinando los valores de transmitancia térmica de cerramientos opacos, carpinterías y cubierta. Se proponen modificaciones al diseño morfológico-funcional y modificaciones en la materialidad de la envolvente, logrando mejoras en el comportamiento de entre $57 \%$ y $68 \%$. Las modificaciones propuestas a la tipología edicilicia, que se repite en el en el $77 \%$ de las escuelas de nivel intermedio, contribuyen a mejorar las condiciones ambientales en estos edificios que no cuentan con sistemas de climatización, mejorando el proceso de enseñanza-aprendizaje y aportando a la reducción del gasto y contaminación en funcionamiento si se implementara el acondicionamiento artificial.

\section{ABSTRACT}

The objective of the work is to analyze the environmental conditions in the classroom spaces of public schools built in the period 2003-2015. The prototypical building, used in $92 \%$ of intermediate level schools, is analyzed, verifying its suitability to the bioclimatic strategies and the thermal behavior of the outer envelope is evaluated, determining the thermal transmittance values of opaque closings of carpentry. Based on the results, modifications are proposed in terms of morphological, spatial and functional design, improving adaptation to passive design strategies and modifications in the materials of the outer envelope, achieving improvements in thermal behavior of between $57 \%$ and $68 \%$. The proposed modifications will have an impact on the area of public construction considering the amount of square meters that are built for school functions, helping to improve the environmental conditions in these buildings, which do not have air conditioning systems.

PALABRAS CLAVE: Espacio aulico, Diagnóstico ambiental, Arquitectura bioambiental.

KEY WORDS: Schools, Environmental analysis, Enviromental architecture.

FECHA DE RECEPCIÓN: 31/3/2021 | FECHA DE ACEPTACIÓN: 25/5/2021

DOI: http://dx.doi.org/10.30972/arq.0174984 
Análisis de condir de clima cálido-húmedo, Tucumán, Argentina

Sandra Gabriela Marquez Vega y Cecilia Fernanda Martinez
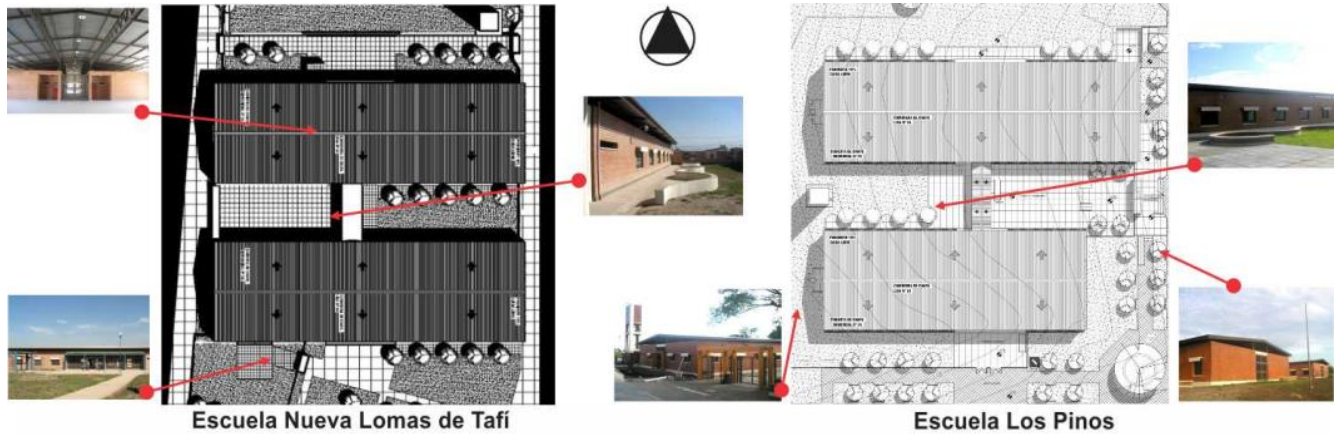

Escuela Los Pinos

Figura 1: Plantas y vistas de prototipos Fuente: Elaboración propia

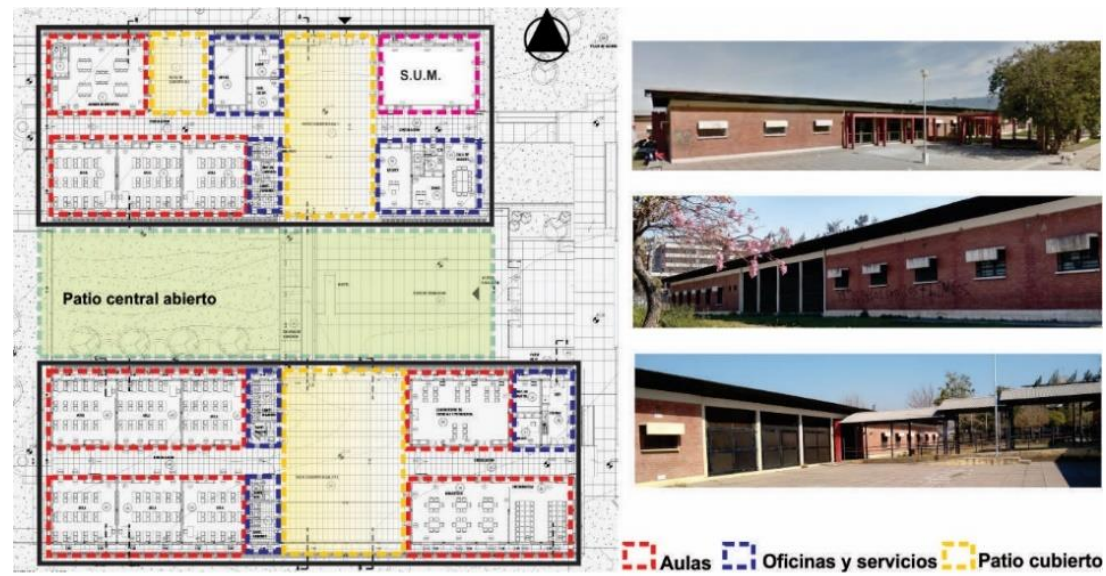

Figura 2: Planta tipo de análisis, con sectores y vistas generales Fuente: Elaboración propia

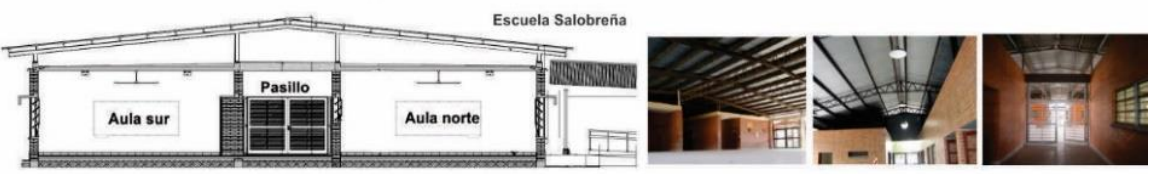

Figura 3: Corte general y vistas interiores de escuela tipo Fuente: Elaboración propia

Los muros las cuatro orientaciones se resuelven igual: ladrillo cerámico visto de $0,27 \mathrm{~m}$ y revoque interior. La cubierta es de losa de hormigón, carpeta alivianada de 0,12 m, membrana hidrófuga y un sobre techo de chapa. Las carpinterías son metálicas pivotantes con vidrio común $3 \mathrm{~mm}$.

\section{Condiciones climáticas y bioclimáticas}

La zona presenta un clima mixto subtropical. Verano cálido-húmedo con temperatura media máxima superior a $32^{\circ} \mathrm{C}$ y humedad relativa media de $85 \%$, vientos $\mathrm{S}$-SO de baja frecuencia y velocidad. Invierno seco y poco riguroso con temperatura media mínima de $6^{\circ} \mathrm{C}$ y humedad relativa media de $65 \%$, vientos S-SO de baja frecuencia y velocidad. Invierno y verano el cielo es semicubierto.

En base a los datos climáticos se realizó un análisis bioclimático y a partir de los resultados obtenidos se establecieron estrategias de base para la zona, figura 4.

El ciclo lectivo, período de uso de las escuelas, comprende de marzo a noviembre, de 8:00 a 18:00 horas. Del diagrama de exigencias bioclimáticas se observa que la necesidad de enfriamiento para verano cubre un $31,5 \%$, ya que el período más caluroso corresponde al receso escolar. La necesidad de calefacción para el período invernal cubre un $20 \%$ del período en los meses de mayo a setiembre, dado que se produce principalmente en horas de la madrugada y la noche. La situación de confort se presenta en un $48,5 \%$ del período escolar. 


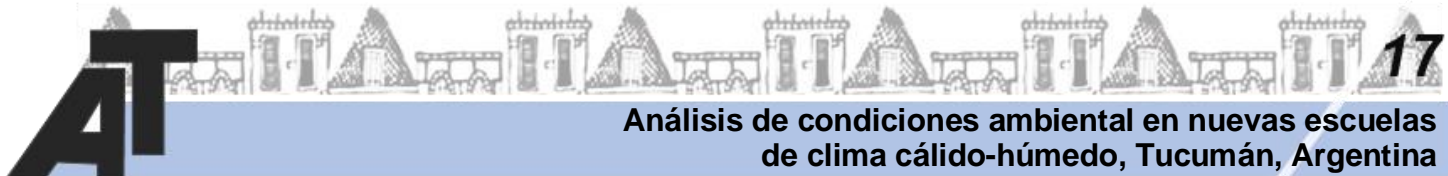
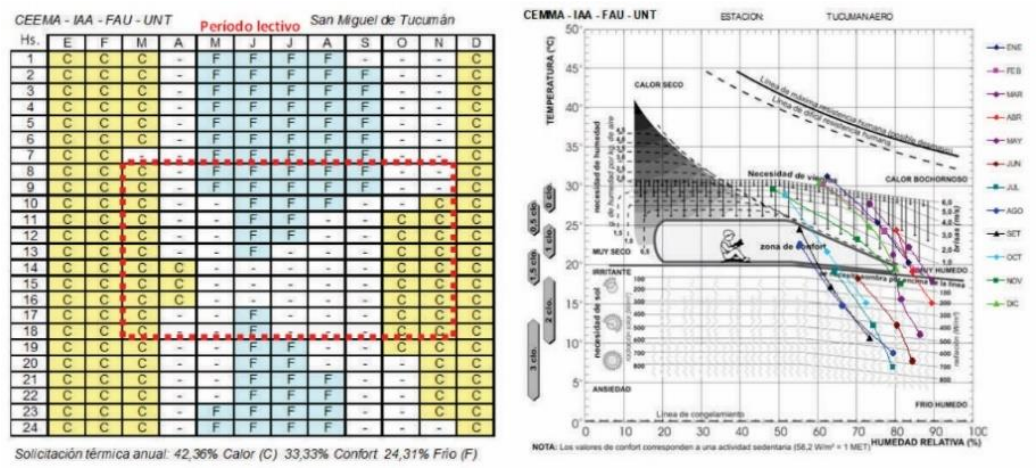

Figura 4: Diagrama Exigencias Bioclimáticas y Gráfica de Olgyay

Del análisis bioclimático se puede establecer que el período de verano se presenta como el más solicitante, aunque no se deben descuidar las necesidades invernales. Siendo así, las principales estrategias a cumplir en verano estarán relacionadas con:

1- el control de la ganancia de calor a través de la envolvente.

2- evitar ingreso de radiación solar directa a través de aberturas.

3- lograr ventilación natural, cruzada y/o forzada.

Para invierno será fundamental evitar las pérdidas de calor a través de la envolvente exterior a fin de mantener el calor generado por ganancias internas, ya que por cuestiones de confort visual no se debe permitir el ingreso de radiación solar directa sobre las áreas de trabajo.

\section{DISCUSIÓN DE RESULTADOS}

Para las evaluaciones ambientales se toman las aulas por ser los espacios de mayor permanencia y ocupación en relación personas $/ \mathrm{m}^{2}$. Si bien el estudio total analizó varios aspectos relacionados a las condiciones ambientales, los más destacados y que tienen mayor influencia como factores en el confort interior son: - ventilación; - asoleamiento; -composición material de la envolvente.

\section{Análisis de ventilación y asoleamiento}

Se analizó la adecuación y respuesta del prototipo a las estrategias bioclimáticas en cuanto a estas dos condicionantes bioclimáticas que se pueden lograr con el adecuado diseño morfológico y espacial del edificio.

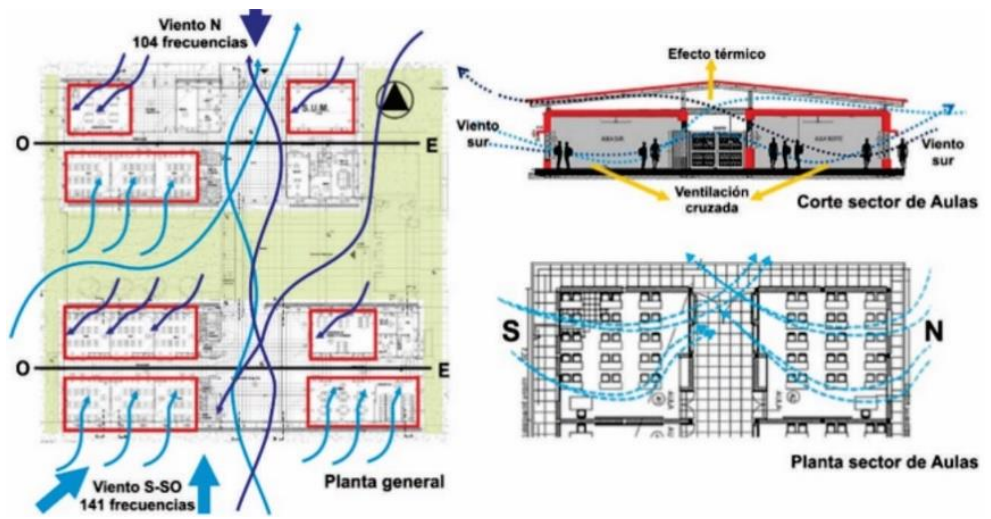

Figura 5: Análisis ventilante del diseño prototípico Fuente: Elaboración propia

Del análisis de ventilación podemos observar, figura 5:

- La planta responde al aprovechamiento de vientos, con la mayor longitud dando a las orientaciones de mayores frecuencias, sur con 141 y norte con 104 (en escala de 1000).

- El esquema de planta abierta permite buena circulación del aire. 
- Las aulas organizadas en tiras con dos frentes libres permite tener caras de presión positiva y negativa, generando la posibilidad de entrada y salida de aire.

- La disposición de aventanamientos enfrentados propicia la ventilación cruzada.

- La relación de superficie de aberturas y superficie de piso del 11,6\%, verifica el valor mínimo de Norma para ventilación higiénica que es del $5 \%$.

Del análisis de asoleamiento podemos observar que:

- La forma alargada del edificio es adecuada, teniendo las superficies mayores a orientaciones de menor exposición de radiación en verano. Esta forma también permite aprovechar la incidencia de radiación en invierno, pero solo en las aulas al con frente norte. Se disponen al norte locales de servicio, administración y aulas especiales, privilegiándolas por sobre las aulas de uso frecuente.

- El patio descubierto entre bloques tiene un tamaño adecuado para permitir la incidencia de radiación directa sobre el frente norte del bloque ubicado al sur.

- Los desbordes principales están orientados al norte lo que permite aprovechar el sol en invierno, pero están muy expuestos en verano por la falta de sistemas de sombreamiento, figura 6 .

- El tipo de protección solar más utilizado es el alero quebrado, en el $53 \%$ de las escuelas. Este se dispone en todas las orientaciones, no adaptándose a las necesidades de sombreado de cada una. - En ventanas, en el $29 \%$ de las escuelas se utiliza un alero horizontal corrido de 0,75 m y en el $18 \%$ un alero horizontal individual de solo $0,30 \mathrm{~m}$. La forma y dimensión de los aleros no resultan eficiente para obstruir totalmente el ingreso de radiación directa, por lo cual se utilizan cortinas interiores para resolver el problema de deslumbramiento sobre las áreas de trabajo, figura 6 .

- Frentes $E$ y $O$ con nula o escasas aberturas reduciendo la ganancia de calor estival.

- El sobre techo ayuda a disminuir la incidencia solar en verano sobre la cubierta, elemento más expuesto, aportando a reducir la ganancia de calor a través de ella.

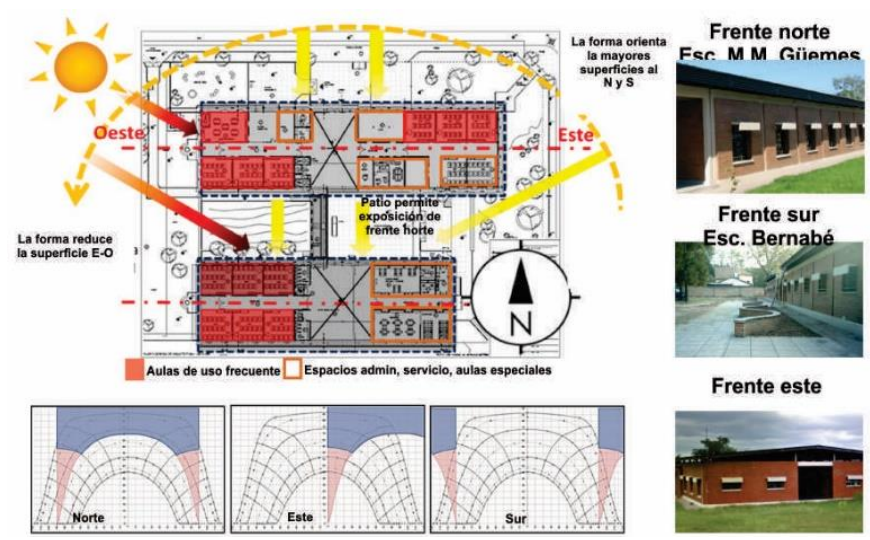

Figura 6: Análisis de asoleamiento Fuente: Elaboración propia

\section{Verificaciones con Normas}

Se verificó el cumplimiento de las recomendaciones de Norma IRAM 11.603 que establece la zonificación bioambiental de la República Argentina, siendo para S. M. de Tucumán la Illb, de clima cálido. Se verificó que solo se cumple adecuadamente con el $33 \%$ de las recomendaciones:

a) Colores claros en exteriores. Esta recomendación no se cumple en los muros de ladrillo cerámico visto en su color natural $(\alpha=0,90)$. Si se cumple para la cubierta de chapa galvanizada $(\alpha=0,5)$.

b) Gran aislación térmica en envolvente al este y al oeste. Esta recomendación no se cumple en el prototipo edilicio ya que ni muros ni cubierta cuentan con aislación térmica.

c) Eje mayor, preferentemente orientado este-oeste. El 92\% de las escuelas consideradas si responden a esta pauta de diseño con sus tiras de aulas en un eje E-O. 


\begin{tabular}{|c|c|c|c|c|}
\hline \multicolumn{5}{|c|}{ Fuente: Elaboración propia } \\
\hline $\begin{array}{l}\text { Componentes de la } \\
\text { envolvente exterior }\end{array}$ & $\begin{array}{c}\text { Superf. } \\
\left(\mathrm{m}^{2}\right)\end{array}$ & $\mathrm{K}\left(\mathrm{W} / \mathrm{m}^{2 \circ} \mathrm{K}\right)$ & \multicolumn{2}{|c|}{$\begin{array}{l}\text { K máximo Norma IRAM } \\
\text { para zona Bio II Nivel C } \\
(\text { mínimo })\left(\mathrm{W} / \mathrm{m}^{20} \mathrm{~K}\right)\end{array}$} \\
\hline Muro & 145,3 & 2,03 & \multirow{2}{*}{$\begin{array}{c}\text { Verano } \\
1,80\end{array}$} & \multirow{2}{*}{$\begin{array}{c}\text { Invierno } \\
1,85\end{array}$} \\
\hline Ventana & 18,8 & 5,70 & & \\
\hline \multicolumn{2}{|c|}{ KMP cerramiento vertical } & 2,45 & NO & NO \\
\hline
\end{tabular}

Tabla 2: Valores de transmitancia térmica media ponderada para muros del prototipo

\section{Propuestas de mejoras}

En base a los resultados obtenidos en los análisis generales y verificaciones de comportamiento térmico se plantean mejoras teniendo en cuenta dos aspectos:

1- Diseño morfológico y funcional.

2- Control solar

3- Materialidad constructiva.

Diseño morfológico y funcional: Considerar las condicionantes climáticas y las estrategias bioclimáticas asociadas son aspecto para considerar en el diseño que no generan costos adicionales de construcción.

Se propone una modificación en la organización de los espacios en la planta prototipo ubicando los bloques de aulas principales hacia el norte y las aulas especiales hacia el sur. El sector administrativo, que en general suele contar con sistemas de climatización se ubica hacia el sur, figura 7.

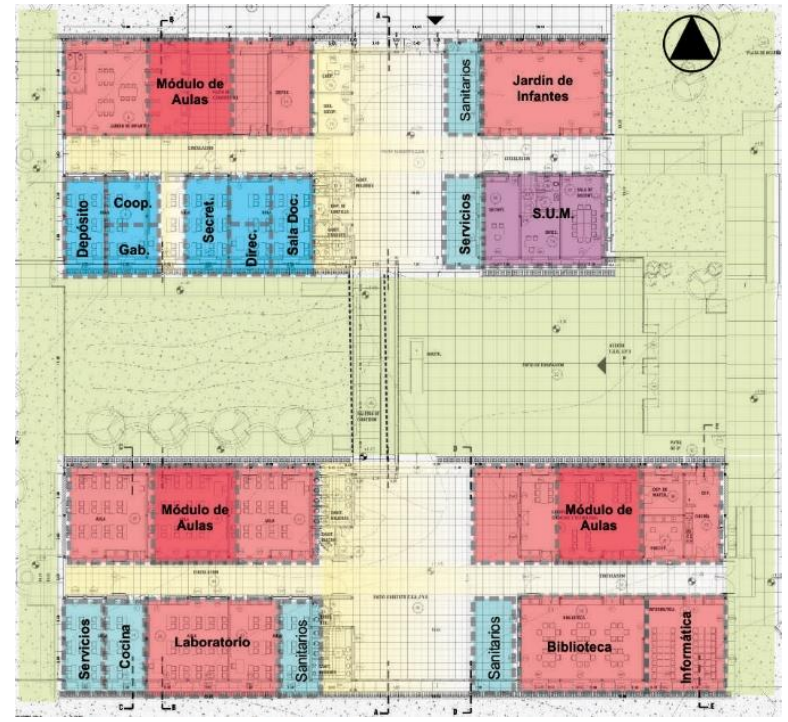

Figura 7: Planta prototípica con modificaciones de distribución propuesta Fuente: Elaboración propia

Control solar: El asoleamiento que reciba un edificio en período estival se puede controlar con sistemas simples a través de disposiciones arquitectónicas y con uso de vegetación.

Para resolver los problemas de incidencia solar directa sobre las aberturas se plantea el uso de una protección exterior más eficiente ya verificada en otros trabajos (Cisterna, Martinez, Márquez 
Análisis de condiciones ambiental en nuevas escuelas de clima cálido-húmedo, Tucumán, Argentina

Sandra Gabriela Marquez Vega y Cecilia Fernanda Martinez

También se recomienda disponer de estructuras que pueden estar alejadas del muro $0.50 \mathrm{~cm}$ permitiendo la ventilación del paramento con plantas trepadoras como: Glisinas, Madre Selvas, Hiedra, Santa Rita, especialmente en los muros orientados al este y oeste.

Materialidad constructiva de la envolvente: Tomando en cuenta los resultados del análisis de comportamiento térmico de la envolvente y las estrategias de "reducción de ganancias y pérdidas de calor a través de la envolvente", se plantea la modificación del tipo de cerramiento exterior, muro y cubierta, y de las carpinterías. Las propuestas para mejora de muros y cubierta pueden aplicarse a las nuevas construcciones, pero también para la rehabilitación de las escuelas ya construidas.

En el caso de la cubierta se propone incorporar aislación térmica de $10 \mathrm{~cm}$ de poliestireno expandido, de uso común en el medio, y un cielorraso de placa de yeso para generar una cámara de aire de $2 \mathrm{~cm}$. Con esta modificación el coeficiente de transmitancia térmica alcanzado verifica para los niveles $\mathrm{B}$ y $\mathrm{C}$ en verano e invierno y para el nivel $\mathrm{A}$ solo en invierno. En el caso de los muros se propone incorporar aislación térmica de poliestireno expandido $0.025 \mathrm{~m}$ y un tabique interior de placas de yeso. Con esto se logra un valor de transmitancia térmica de $0,78 \mathrm{~W} / \mathrm{m}^{2} \mathrm{~K}$, verificando con nivel $\mathrm{C}$ y $\mathrm{B}$ de transmitancia máxima para verano e invierno, tabla 3.

Fuente: Elaboración propia

\begin{tabular}{|c|c|c|c|c|c|c|c|c|c|c|c|c|c|c|c|}
\hline \multirow{2}{*}{\multicolumn{2}{|c|}{$\begin{array}{l}\text { Cubierta } \\
\text { propuesta }\end{array}$}} & \multicolumn{6}{|c|}{$\begin{array}{l}\text { K máximo Norma IRAM para zona Bio II (temp. } \\
\text { mín. diseño }-0,20^{\circ} \mathrm{C} \text { ) }\end{array}$} & & & \multicolumn{6}{|c|}{$\begin{array}{l}\text { K máximo Norma IRAM para zona Bio II (temp. } \\
\text { mín. diseño }-0,20^{\circ} \mathrm{C} \text { ) }\end{array}$} \\
\hline & & \multicolumn{2}{|c|}{$\begin{array}{c}\text { Nivel C } \\
\text { (mínimo) }\end{array}$} & \multicolumn{2}{|c|}{$\begin{array}{c}\text { Nivel B } \\
\text { (recomendado) }\end{array}$} & \multicolumn{2}{|c|}{$\begin{array}{l}\text { Nivel A } \\
\text { (ecológico) }\end{array}$} & \multirow{2}{*}{\multicolumn{2}{|c|}{ Muro propuesto }} & \multicolumn{2}{|c|}{$\begin{array}{c}\text { Nivel C } \\
\text { (mínimo) }\end{array}$} & \multicolumn{2}{|c|}{$\begin{array}{c}\text { Nivel B } \\
\text { (recomendado) }\end{array}$} & \multicolumn{2}{|c|}{$\begin{array}{l}\text { Nivel A } \\
\text { (ecológico) }\end{array}$} \\
\hline $\mathrm{K}(\mathrm{W})$ & $\left.{ }^{\circ} \mathrm{K}\right)$ & $\begin{array}{l}\text { Ver. } \\
0,72\end{array}$ & $\begin{array}{l}\text { Inv. } \\
1,00\end{array}$ & $\begin{array}{l}\text { Ver. } \\
0,45\end{array}$ & $\begin{array}{l}\text { Inv. } \\
0,83\end{array}$ & $\begin{array}{l}\text { Ver. } \\
0,18\end{array}$ & $\begin{array}{l}\text { Inv. } \\
0,32\end{array}$ & & & $\begin{array}{l}\text { Ver. } \\
1,80\end{array}$ & $\begin{array}{l}\text { Inv. } \\
1,85\end{array}$ & $\begin{array}{l}\text { Ver. } \\
1,10\end{array}$ & $\begin{array}{l}\text { Inv. } \\
1,00\end{array}$ & $\begin{array}{l}\text { Ver. } \\
0,45\end{array}$ & $\begin{array}{l}\text { Inv. } \\
0,38\end{array}$ \\
\hline Verano & 0,23 & SI & SI & SI & SI & NO & SI & $\begin{array}{l}\text { K ver./inv. } \\
\left.\text { (W/M2 }{ }^{\circ} \mathrm{K}\right)\end{array}$ & 0,78 & SI & SI & SI & SI & No & NO \\
\hline
\end{tabular}

\begin{tabular}{|l|c|c|c|c|c|c|c|}
\hline Invierno & 0,24 & $\mathrm{SI}$ & $\mathrm{SI}$ & $\mathrm{SI}$ & $\mathrm{SI}$ & $\mathrm{NO}$ & $\mathrm{SI}$ \\
\hline
\end{tabular}

Tabla 3: Valores de transmitancia térmica de la envolvente propuesta

Para las carpinterías se propone el uso de DVH con vidrio común, ( $3 \mathrm{~mm}$ - CA $6 \mathrm{~mm}-3 \mathrm{~mm}$ ), con una transmitancia térmica de $3,20 \mathrm{~W} / \mathrm{m}^{20} \mathrm{~K}$, calificando en categoría $\mathrm{K} 5$ de aislación térmica.

Con los valores de transmitancia térmica de la envolvente exterior propuesta para muros y carpinterías se logra que el valor medio ponderado del cerramiento vertical verifique con el valor máximo admisible para Nivel C, e incluso para el nivel B en condición de invierno, tabla 4.

Fuente Elaboración propia

\begin{tabular}{|c|c|c|c|c|c|c|}
\hline \multirow{3}{*}{$\begin{array}{c}\text { Componentes de la } \\
\text { envolvente exterior } \\
\text { Muro }\end{array}$} & \multirow{3}{*}{$\begin{array}{c}\text { Superf. }\left(\mathrm{m}^{2}\right) \\
145,3\end{array}$} & \multirow{3}{*}{$\begin{array}{c}\begin{array}{c}\mathrm{K} \\
\left(\mathrm{W} / \mathrm{m} 2^{\circ} \mathrm{K}\right)\end{array} \\
0,78\end{array}$} & \multicolumn{4}{|c|}{$\begin{array}{l}\text { K máximo Norma IRAM zona Bio II } \\
\left(\mathrm{W} / \mathrm{m} 2^{\circ} \mathrm{K}\right)\end{array}$} \\
\hline & & & \multicolumn{2}{|c|}{$\begin{array}{l}\text { Nivel C } \\
\text { (mínimo) }\end{array}$} & \multicolumn{2}{|c|}{$\begin{array}{c}\text { Nivel B } \\
\text { (recomendado) }\end{array}$} \\
\hline & & & Verano & Invierno & Verano & Invierno \\
\hline Ventana & 18,8 & 3,20 & 1,80 & 1,85 & 1,10 & 1,00 \\
\hline \multicolumn{2}{|c|}{ KMP cerramiento vertical } & 1,05 & $\mathrm{SI}$ & SI & $\mathrm{SI}$ & NO \\
\hline
\end{tabular}

Tabla 4: Valores de transmitancia térmica media ponderada para componentes modificados

\section{CONCLUSIONES}

En un contexto sociopolítico y económico en el cual no es factible pensar en proveer de acondicionamiento artificial a las escuelas pública, es fundamental que los edificios logren las condiciones ambientales adecuadas con estrategias pasivas a través de un adecuado diseño morfológico, uso de materiales y diseño de protecciones solares. 
